\section{NAPCRG ANNUAL MEETING DISTINGUISHED PAPERS}

NAPCRG's Annual Meeting is a forum for primary care researchers from across the globe to gather and present their research, collaborate for new research, and foster growth for up-and-coming researchers. The 2013 Annual Meeting will be held in Ottawa, Ontario, November 9-13, 2013.

Three papers were selected from all submissions and given the special designation of "distinguished paper." The distinguished papers highlight excellence in research and contain all of the following factors: overall excellence, quality of research methods, quality of the writing, relevance to primary care clinical research, and overall impact of the research on primary care and/or clinical practice.

Below are brief summaries of this year's distinguished papers.

\section{Involving the Public in Setting Priorities for Primary Healthcare Improvement: A Cluster Randomized Trial}

(Oral Presentation On Completed Research Full Paper)

Antoine Boivin, $\mathrm{MD}, \mathrm{PhD}$, Université de Sherbrooke;

Pascale Lehoux, $\mathrm{PhD}_{i}$ Réal Lacombe, $\mathrm{MD}, \mathrm{MPH}_{i}$ Jako

Burgers, $\mathrm{MD}, \mathrm{PhD}_{i}$ Richard Grol, $\mathrm{PhD}$

Patients are increasingly involved in collective decision making about primary health care, including practice redesign, patient-centered medical home demonstration projects, clinical guidelines, and quality improvement activities. However, research has remained focused on patients' individual roles and provides little guidance as to how they can be effectively involved in collective health care choices. This paper reports on the first trial of patient involvement in health care improvement priority setting. Patient involvement changed health care improvement priorities toward access to primary care, self-care support, and community partnership $(P<0.05)$. Conversely, priorities established by professionals alone remained at odds with patients' priorities and were more focused on emergency room visits and the technical quality of single disease management. The results of this study are important for primary care clinicians and researchers, as they demonstrate the feasibility and impact of involving patients together with professionals in setting priorities driving health care improvement efforts.

\section{Will Rural Patients Benefit From the Patient- Centered Medical Home? A Card-Study in the High Plains Research Network}

(Oral Presentation On Completed Research Full Paper)

John Westfall, MD, MPH, University of Colorado; Linda Zittleman, $\mathrm{MSPH}_{i}$ Marc Ringel, $\mathrm{MD}_{i}$ Christin Sutter, BA, Kelly McCaffrey, BA; Tony Gerdt, MD; Sergio Sanchez, BA; William Leblanc, BA; Susan Gale, $\mathrm{MSPH}_{i}$ Perry Dickinson, MD

The Patient-Centered Medical Home (PCMH) has become a dominant model for improving the quality and cost of primary care in the United States. A frequent requirement for enhanced reimbursement from Medicare and other insurance companies includes formal certification of 21 practice level activities. If the $\mathrm{PCMH}$ is to improve patient outcomes and decrease cost, practices will need to incorporate components of the PCMH into their day-to-day care of patients. However, it is unclear whether providers actually perceive patient benefit from all components of the $\mathrm{PCMH}$. Rural providers face a unique set of challenges to implementing all aspects of the PCMH including geographic isolation, small populations, privacy concerns, and limited high-speed connectivity. Given these concerns, the High Plains Research Network sought to determine the perceived patient benefit for 18 core components of the PCMH.

Thirty-six practices participated in a 1-day "cardstudy" research project. Each provider completed a card for each patient. After several brief demographic questions, the provider was asked, "Which features of a patient-centered medical home do you believe did or would have benefitted this patient?" Seventy-eight providers in 36 practices completed over 1,000 individual patient cards. The primary reason for the visit was $45 \%$ for acute visit, $35 \%$ for chronic visit, and $20 \%$ for a health maintenance visit. There was wide variation among the perceived benefits of the $18 \mathrm{PCMH}$ components ranging from just $8 \%$ for the lowest ranked PCMH component to over $65 \%$ for the highest. There were significant differences in perceived benefit between visits for acute and chronic care.

There may be current required PCMH elements that should no longer be required for rural practices, and could be elective or ancillary PCMH elements. Certainly, a provider who perceives no benefit to a $\mathrm{PCMH}$ element is unlikely to incorporate it into usual patient care. Conversely, PCMH practice coaching and educational efforts may address perceived benefit and provide additional rationale for implementing some PCMH elements. 


\section{Targeted and Tailored Patient Engagement Interventions to Enhance Recognition and Initial Treatment of Depression in Primary Care: Randomized Controlled Trial}

(Oral Presentation On Completed Research Full Paper)

Anthony Jerant, MD, University of California-Davis Health System; Peter Franks, MD; Daniel Tancredi, PhD; Mitchell Feldman, MD, MPhil; Christina Slee,

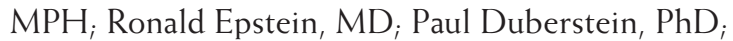
Robert Bell, PhD; Maja Jackson-Triche, MD, MSPH; Debora Paterniti, PhD, Richard Kravitz, MD, MSPH Primary care interventions to encourage patients to disclose depressive symptoms and accept initial depression treatment hold promise for improving depression care quality. The objective of this study was to determine whether a targeted public service announcement (PSA) or a tailored interactive multimedia computer program (IMCP), both delivered in primary care offices immediately before provider visits, can increase patient engagement in care and improve initial depression care (IDC).

This randomized controlled trial consisted of 559 working-age adults stratified by depression symptom burden (Patient Health Questionnaire-9 [PHQ-9] score 5-9 [mild] or $\geq 10$ [moderate or greater]). Interventions used were (1) A depression PSA, targeted to gender and socioeconomic status; (2) an IMCP, individually tailored to depression-related symptoms, beliefs, and visit agenda, or (3) an attention control video. The primary outcome measure was the composite measure of IDC (depression medication prescription, mental health referral, or both) and the secondary measure was patient-reported requests for depressionrelated information, self-efficacy for communicating with providers about mental health, and 12-week depression symptoms and health status.

Compared with control, the IMCP (but not the PSA) was associated with greater delivery of IDC (adjusted odds ratio [AOR] 1.81, 95\% confidence interval [CI] $1.04,3.16)$. In analyses stratified by depression symptom burden, the IMCP effect on IDC was statistically significant only among patients with at least moderate symptoms (AOR 2.42, 95\% CI 1.11, 5.30). Both PSA (AOR $2.11,95 \%$ CI 1.12, 3.98) and IMCP (AOR 2.19, 95\% CI $1.19,4.04)$ patients were more likely than controls to request depression-related information. Neither intervention significantly affected 12 -week outcomes.

Both the targeted PSA and tailored IMCP successfully encouraged patients to request depression-related information from providers. Only the IMCP was associated with greater delivery of initial depression care. Tailored IMCPs can help patients become agents for improving the quality of their own care.

Kristin Robinson, NAPCRG
From the Agency for Healthcare

Research and Quality

\section{AHRQ UPDATES ON PRIMARY CARE RESEARCH: CLINICAL-COMMUNITY RELATIONSHIPS}

Sustainable, effective relationships among primary care clinicians and community organizations that provide preventive services have the potential to improve patient access to evidence-based clinical preventive services and, ultimately, to improve the health of people and their communities.

The Agency for Healthcare Research and Quality (AHRQ) has been working to promote collaborations among primary care practices, public health departments, and community organizations through its Clinical-Community Relationships initiative. AHRQ is exploring how these collaborative relationships and partnerships are developed, strengthened, and sustained to improve care and meet the needs of patients and families.

AHRQ's work in this area began in 2008 with the convening of a Clinical-Community Linkages Summit, which was designed to encourage collaboration among key clinical-community stakeholders. A literature review and the development of clinical-community relationship case studies followed in an effort to build an evidence base for this approach. In 2010, AHRQ convened a second Clinical-Community Relationships summit to develop a national strategy for promoting these relationships. ${ }^{1}$

Since then, AHRQ has moved forward on several fronts to address the research needs identified at the 2010 conference:

\section{Clinical Community Relationships Measures (CCRM) Atlas}

AHRQ created the Atlas, published in March 2013, to identify ways to define, measure, and evaluate programs that use clinical-community relationships to deliver clinical preventive services. ${ }^{2}$ The Atlas includes 22 measures of structure, process, and outcomes that are within a clinical-community relationships measurement framework. The Atlas also describes and illustrates how the measures can be directly implemented by evaluators. ${ }^{3}$

http://www.ahrq.gov/professionals/preventionchronic-care/resources/clinical-communityrelationships-measures-atlas/index.html 